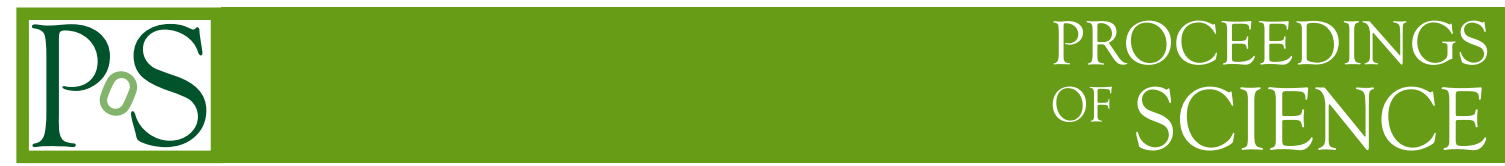

\title{
Gamma-ray variability of radio-loud narrow-line Seyfert 1 galaxies
}

\section{G. Calderone*}

Università di Milano - Bicocca, Dip. di Fisica G. Occhialini, Piazza della Scienza 3, I-20126

Milano, Italy

E-mail: giorgio.calderone@mib.infn.it

The variability of $\gamma$-ray emission from radio-loud narrow-line Seyfert 1 galaxies has been recently confirmed on a rather firm statistical basis [6]. In this work we extend the variability analysis by including data from Fermi/LAT up to june 2011 and by using the latest release of the ScienceTools software package and of the LAT instrument response function.

Narrow-Line Seyfert 1 Galaxies and their place in the Universe - NLS1,

April 04-06, 2011

Milan Italy

\footnotetext{
* Speaker.
} 


\section{Introduction}

Narrow-line Seyfert 1 galaxies are a peculiar class of Type 1 AGN which shows unusually narrow permitted Balmer lines with FWHM $\lesssim 2000 \mathrm{~km} \mathrm{~s}^{-1}$, as well as a strong FeII bump, a soft $\mathrm{X}$-ray excess and flux ratio [OIII]/H $\beta<3[12,13]$. These sources are usually weak radio emitters, although few of them $(\sim 7 \%)$ are formally radio-loud $[10,14]$. Such sources are dubbed radioloud narrow-line Seyfert 1 galaxies (RL-NLS1). Recently it has been shown that some radio-loud narrow-line Seyfert 1 galaxies are also $\gamma$-ray emitters [7, 1, 2, 3]. The radio properties of RL-NLS1 (namely variability, flat spectrum and high brightness temperature) suggests some similarities with blazars, i.e. the presence of a relativstic jet closely aligned to the line of sight, as speculated by several authors $[16,10,15,14,8,9]$. The detection of $\gamma$-ray emission from these sources goes exactly in this direction.

An important issue about $\gamma$-ray detection of RL-NLS1 galaxies is the variability of the emission. Steady $\gamma$-ray emission may be due to an intense star formation in the host galaxy [4], thus detection of variability allows us to exclude a starburst activity as the main origin of the $\gamma$-ray emission from RL-NLS1. Furthermore, the measure of a variability timescale allow to estimate an upper limit to the size of the region where $\gamma$-ray photons are emitted. These studies have been undertaken by Calderone et al. 2011 [6] (hereafter C11) using data from Fermi/LAT for the four

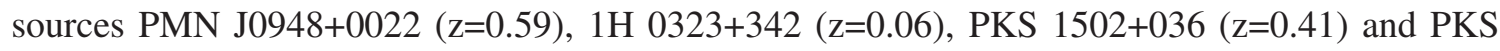
2004-447 ( $\mathrm{z}=0.24)$. It turned out that the $\gamma$-ray emission from the four analyzed RL-NLS1 is actually variable with a high level of significance and that the minimum variability timescale lie in the range 3 - 30 days. In this work we repeat the same analysis performed in $\mathrm{C} 11$, but using data covering a longer period, as well as the latest versions of the ScienceTools software package and of the instrument response function released by the LAT team.

\section{Data analysis}

Here we will briefly review the data analysis process, for a deeper discussion see C11 and references therein. The ScienceTools software version used in this work is 9.23.1 and the LAT Instrument Response Function (IRF) is P6_V11. Analyzed data span a period of 34 months, from august 2008 to june 2011 and covers a Regions Of Interest (ROI) of $10^{\circ}$ around each of the four source's catalog positions. The unbinned likelihood data analysis has been performed following the standard procedures described in the Fermi/LAT documentation: we modeled each source in the LAT 1-year point source catalog [5] inside the ROI with a power law in the range $0.1-100 \mathrm{GeV}$. We extracted light curves using time bins of 15 days (Fig. 1; time binning for PKS 2004-447 is 30 days) using a TS threshold of 10 (TS $>10$, roughly equivalent to $3 \sigma$, [11]). For non-significant detection $(\mathrm{TS}<10)$ we computed an upper limit to the flux by varying the source flux value (obtained through maximization of likelihood) until TS reaches a value of 4 [5]. Resulting fluxes corresponds to $2 \sigma$ upper limits, and are denoted with arrows in the figure. If TS $<1$ we didn't compute the upper limit since it would be overestimated. Then we performed a chi-squared test against the null hypotesis of constant flux equal to a reference value. In $\mathrm{C} 11$ we used the flux estimate obtained with a single likelihood analysis over the entire period of $\sim 26$ months as reference value. In this work we use a reference flux equal to the weighted mean of the fluxes measured in each time bin, thus we expect 

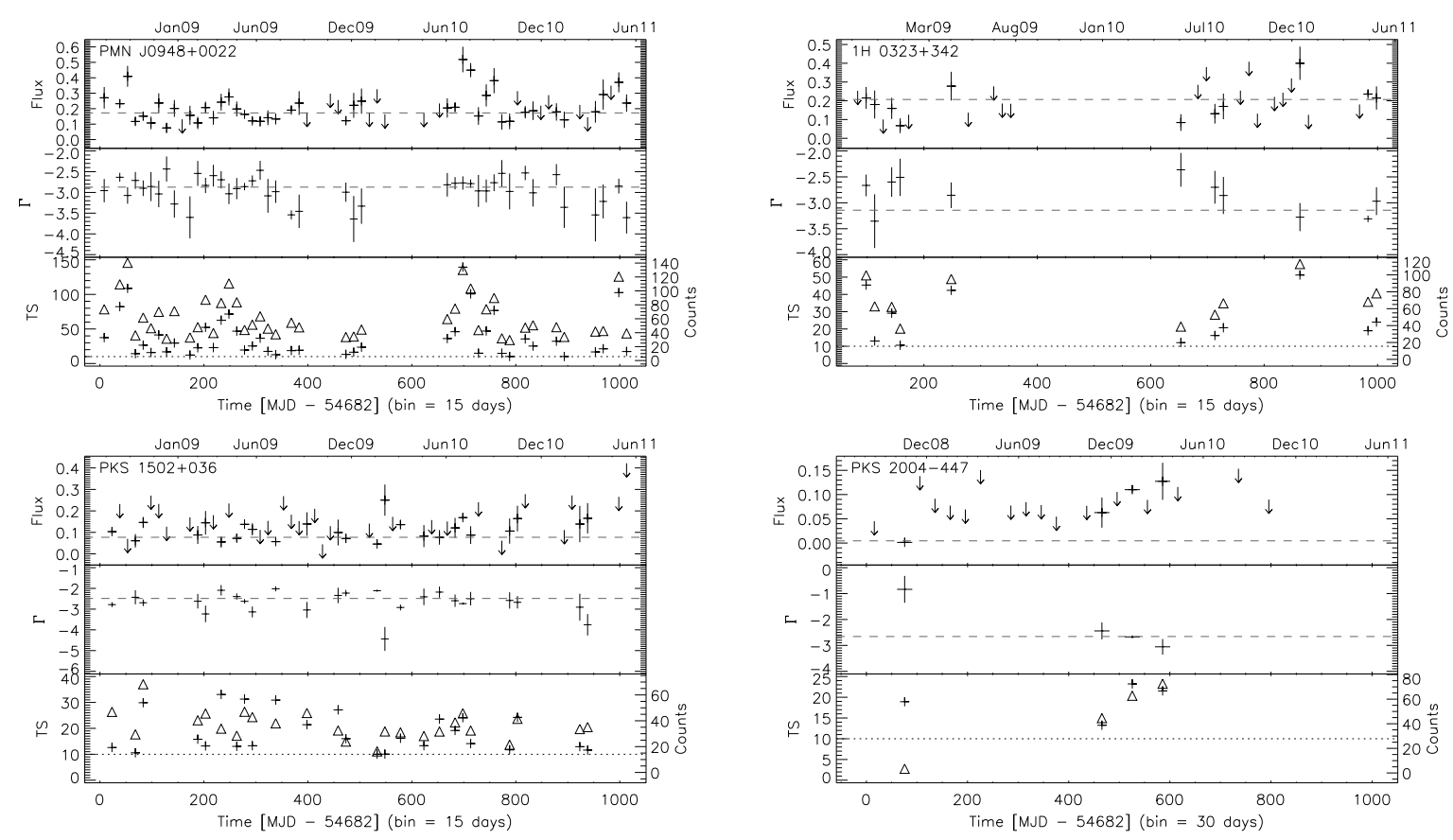

Figure 1: Upper panels show light curves for detections with $\mathrm{TS}>10$. Flux units are $10^{-6} \mathrm{ph} \mathrm{cm}^{-2} \mathrm{~s}^{-1}$ in the range $0.1-100 \mathrm{GeV}$. Vertical error bars correspond to $1 \sigma$ errors, horizontal bars corresponds to the time binning (15 days for PMN J0948+0022, 1H 0323+342 and PKS 1502+036, 30 days for PKS 2004-447). Upper limits $(\mathrm{TS}<10)$ denoted by arrows are given $2 \sigma$ significance level. Middle panels: photon indices. Vertical error bars correspond to $1 \sigma$ errors. Horizontal dashed lines in upper and middle panels are the weighted mean flux over the period of 34 months and weigthed mean photon index respectively. Lower panels: TS values (plus symbols) and number of counts associated to the analyzed source (triangle symbols, values on the right axis).

to find a smaller value of $\chi^{2}$. Furthermore, in C11 the minimum binning interval was $\sim 6$ hours, while in this work is $\sim 1$ day. Results are given in Tab. 2 (col. 7 and 8).

To estimate a minimum variability timescale we further proceed in analyzing data by recursively halving the time bin interval for each time bin with significant detection (TS $>10)$. Then, we considered all combinations of non-overlapping time bins having a significant detection in both time bins, a flux difference greater than the greatest flux error involved at the $3 \sigma$ level, a count difference significant at the $3 \sigma$ level (assuming a Poisson statistic) and number of counts greater than 3 in both bins. For such pairs of bins we computed the $e$-folding timescale (that is the time needed to change the flux by a factor $\sim 2.7$ ) and took the smaller value as the minimum variability timescale $\tau$. Finally, we computed the associated error by error propagation (Eq. 1 and 2 in C11). Results are given in Tab. 2 (col. 9). Quantities involved in calculation of $\tau$ and $\Delta \tau$ are given in Tab. 2 .

\section{Discussion and conclusions}

We used data from Fermi/LAT in order to assess the variability of $\gamma$-ray emission of four RLNLS1. The same analysis has been performed in C11 [6], the only differences being: the length 


\begin{tabular}{lccr|rrrrrr}
\hline \multicolumn{1}{c}{ Source } & $\mathrm{z}$ & $\begin{array}{c}\mathrm{D}_{\mathrm{L}} \\
{[\mathrm{Gpc}]}\end{array}$ & \multicolumn{2}{c}{$\begin{array}{c}\mathrm{L}_{\gamma} \\
{\left[10^{45} \mathrm{erg} \mathrm{s}^{-1}\right]}\end{array}$} & \multicolumn{1}{c}{$\chi^{2}$} & $\mathrm{DOF}$ & \multicolumn{1}{c}{$\begin{array}{c}\tau \\
{[\text { days] }}\end{array}$} \\
\hline PMN J0948+0022 & 0.59 & 3.40 & 1000 & 308.00 & -2.872 & 148 & 42 & $-4.6 \pm 3.3$ \\
1H 0323+342 & 0.06 & 0.27 & 151 & 0.63 & -3.143 & 34 & 10 & $1.3 \pm 0.7$ \\
PKS 1502+036 & 0.41 & 2.20 & 1549 & 49.57 & -2.486 & 201 & 24 & $53.5 \pm 50.6$ \\
PKS 2004-447 & 0.24 & 1.20 & 6320 & 0.55 & -2.657 & 240 & 3 & $86.5 \pm 44.4$ \\
\hline
\end{tabular}

Table 1: Data and results of the analysis on the four RL-NLS1 sources. Columns are: (1) name of the source; (2) redshift; (3) luminosity distance; (4) radio loudness; (5) $\gamma$-ray luminosity $(0.1-100 \mathrm{GeV})$ corresponding to the weighted mean of fluxes in the light curves; (6) weighted mean of photon indices; (7) $\chi^{2}$ and (8) DOF computed on the light curves in the null hypotesis of constant flux equal to the weighted mean flux; (9) minimum $e$-folding variability timescale with error at $3 \sigma$ level.

\begin{tabular}{crrcrr}
\hline Source & $\begin{array}{c}t^{1} \\
\text { [days] }\end{array}$ & $\begin{array}{c}\Delta t^{2} \\
\text { [days] }\end{array}$ & $\begin{array}{c}\mathrm{F}_{\gamma}^{3} \\
{\left[10^{-6} \mathrm{ph} \mathrm{cm}^{-2} \mathrm{~s}^{-1}\right]}\end{array}$ & $\begin{array}{c}\mathrm{N} / \Delta t \\
\text { [cts days }\end{array}$ & $\mathrm{TS}$ \\
\hline PMN J0948+0022 & 709.40 & 7.50 & $0.406 \pm 0.015$ & 6.9 & 54 \\
& 703.30 & 0.94 & $1.510 \pm 0.215$ & 29.7 & 58 \\
1H 0323+342 & 251.00 & 1.88 & $0.270 \pm 0.054$ & 7.9 & 10 \\
& 253.30 & 0.94 & $1.569 \pm 0.065$ & 33.9 & 36 \\
PKS 1502+036 & 255.70 & 30.00 & $0.085 \pm 0.032$ & 2.0 & 16 \\
& 334.40 & 7.50 & $0.369 \pm 0.079$ & 5.6 & 22 \\
PKS 2004-447 & 75.66 & 30.00 & $0.001 \pm 0.001$ & 0.1 & 19 \\
& 527.50 & 3.75 & $0.264 \pm 0.043$ & 4.3 & 12 \\
\hline
\end{tabular}

Table 2: Quantities involved in the computation of the minimum $e$-folding variability timescale.
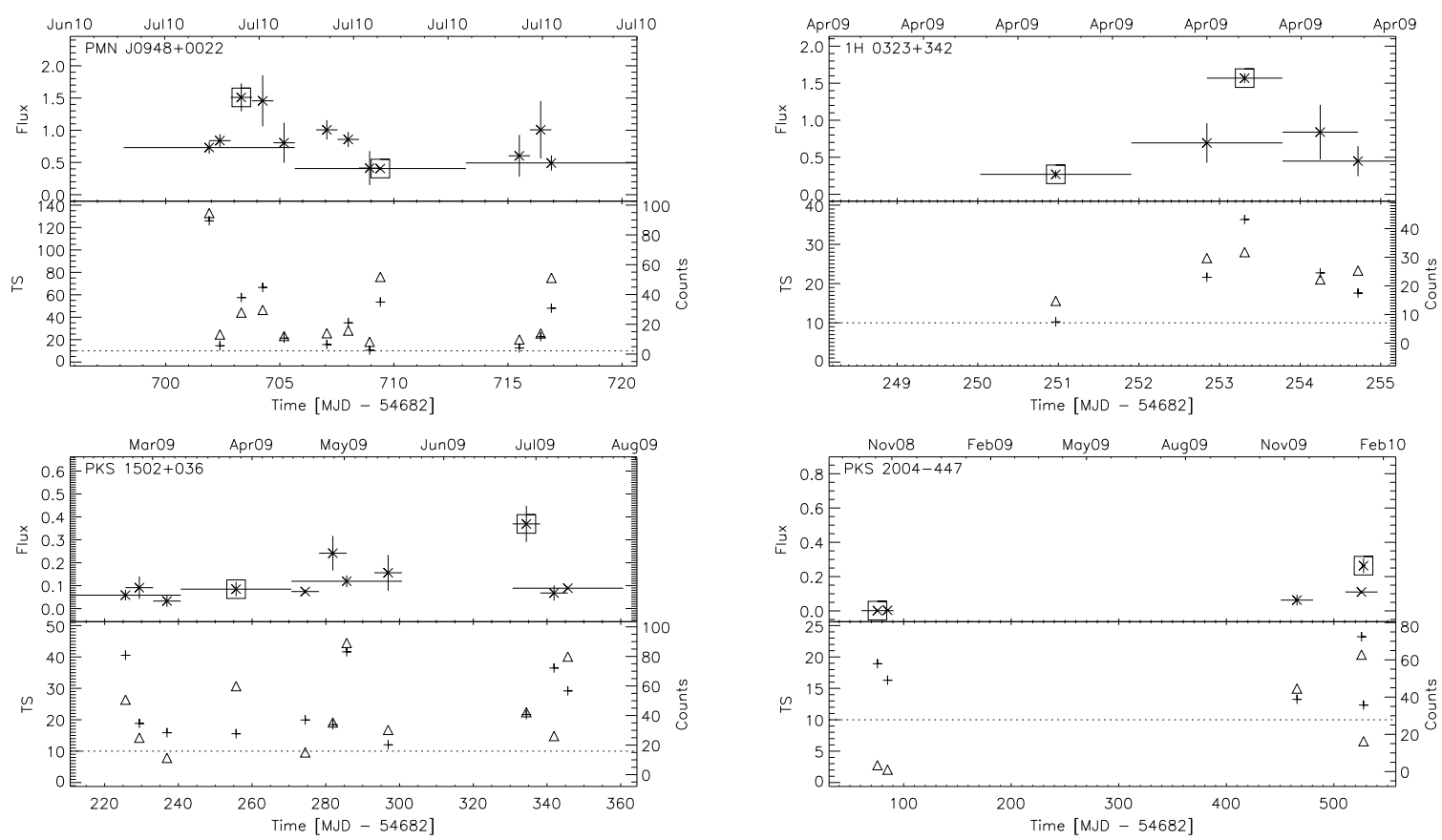

Figure 2: Detailed view on the bins (denoted by squares) involved in the computation of the minimum variability timescale. Units and meaning of symbols are the same as in Fig. 1 (upper and lower panels). Flux symbols have been changed to $\times$ for a clearer visibility. See also Tab. 2 . 
of the period of analysis ( $\sim 34$ months vs. $\sim 26$ months), the version of the ScienceTools software package $^{4}$ (9.23.1 vs. 9.15.2), the LAT instrument response function (P6_V11 vs. P6_V3), the reference flux used in calculation of $\chi^{2}$ (weighted mean of fluxes in light curve vs. flux computed in a single likelihood analysis covering the entire period), the minimum time binning interval $(\sim 1$ day vs. $\sim 6$ hours). A statistically significant variability over the period of $\sim 34$ months is present for all sources, although with a lower level of significance with respect to C11. This is mainly due to the choice of using the weighted mean of fluxes in the light curve as a reference, that is the value which minimizes the $\chi^{2}$ by definition. Also, the new flux estimates show slightly greater errors than with previous releases of software and IRF, resulting in lower values of $\chi^{2}$ and greater estimates of minimum variability timescales with respect to the result given in $\mathrm{C} 11$. An important exception is the minimum variability timescale for the source $1 \mathrm{H} 0323+342$, which is significantly smaller in this work rather than in C11. This is because one of the bin involved in calculation has a TS value which is slightly greater than the threshold (see Tab. 2), while it was below the threshold in $\mathrm{C} 11$, thus it was excluded from our calculation. This example clearly highlights that our estimates of minimum variability timescale should actually be considered as upper limits, rather than measures, of the intrinsic variability timescale of the source. The case of $1 \mathrm{H} 0323+342$, as well as PMN J0948+0022 in this work and C11, clearly shows that at least two RL-NLS1 are able to change their $\gamma$-ray flux significantly on timescales on the order of few days. This allows to set an upper limit to the size of the emitting region $R_{\text {blob }} \lesssim \delta c \tau /(1+z) \sim 0.8-18 \delta_{1} \times 10^{17} \mathrm{~cm}$, where $\delta_{1}=\delta / 10$ is the relativistic Doppler factor and $z$ is the redshift. Thus we can rule out the possibility that the $\gamma$-ray emission is due to an intense star formation rate in the host galaxy, and support the hypotesis of the presence of a jet closely aligned to the line of sight.

In summary, the results of $\mathrm{C} 11$ are confirmed in this work, where we used the new version of the software package and of the LAT instrument response function. The significance of the variability and the minimum variability timescales clearly depends on the way they are measured, but in any case we can be confident that the RL-NLS1 sources analyzed here are actually variable $\gamma$-ray emitters and that the minimum observed timescales are of the order of a few days for at least two sources.

\section{References}

[1] A. A. Abdo et al. (Fermi/LAT Collaboration): Fermi/Large Area Telescope Discovery of Gamma-Ray Emission from a Relativistic Jet in the Narrow-Line Quasar PMN J0948+0022. ApJ, 699 (2009) 976.

[2] A. A. Abdo et al. (Fermi/LAT Collaboration): Multiwavelength Monitoring of the Enigmatic Narrow-Line Seyfert 1 PMN J0948+0022 in 2009 March-July. ApJ, 707 (2009) 727.

[3] A. A. Abdo et al. (Fermi/LAT Collaboration): Radio-Loud Narrow-Line Seyfert 1 as a New Class of Gamma-Ray Active Galactic Nuclei. ApJ, 707 (2009) L142.

[4] A. A. Abdo et al. (Fermi/LAT Collaboration): Detection of Gamma-Ray Emission from the Starburst Galaxies M82 and NGC 253 with the Large Area Telescope on Fermi. ApJ, 709 (2010) L152.

[5] A. A. Abdo et al. (Fermi/LAT Collaboration): Fermi Large Area Telescope First Source Catalog. ApJS, 188 (2010) 405.

\footnotetext{
${ }^{4}$ The discrepancies in results between versions are statistically compatible with zero, although there may be significant differences for low values of TS.
} 
[6] G. Calderone et al.: $\gamma$-ray variability of radio-loud narrow-line Seyfert 1 galaxies. MNRAS, 413 (2011) 2365.

[7] L. Foschini et al.: Fermi/LAT discovery of gamma-ray emission from a relativistic jet in the narrow-line Seyfert 1 quasar PMN J0948+0022. In: Accretion and Ejection in AGNs: a global view, L. Maraschi et al. eds, ASP Conference Series 427 (2010) 243.

[8] L. Foschini et al.: Blazar nuclei in radio-loud narrow-line Seyfert 1? Advances in Space Research, 43 (2009) 889.

[9] M. Gu and Y. Chen: The Compact Radio Structure of Radio-loud Narrow Line Seyfert 1 Galaxies. AJ, 139 (2010) 2612.

[10] S. Komossa et al.: Radio-loud Narrow-Line Type 1 Quasars. AJ, 132 (2006) 531.

[11] J. R. Mattox et al.: The Likelihood Analysis of EGRET Data. ApJ, 461 (1996) 396.

[12] D. E. Osterbrock and R. W. Pogge: The spectra of narrow-line Seyfert 1 galaxies. ApJ, 297 (1985) 166.

[13] R. W. Pogge: Narrow-line Seyfert 1s: 15 years later. New Astronomy Review, 44 (2000) 381.

[14] W. Yuan et al.: A Population of Radio-Loud Narrow-Line Seyfert 1 Galaxies with Blazar-Like Properties? ApJ, 685 (2008) 801.

[15] H. Zhou et al.: A Narrow-Line Seyfert 1-Blazar Composite Nucleus in 2MASX J0324+3410. ApJ, 658 (2007) L13.

[16] H.-Y. Zhou et al.: SDSS J094857.3+002225: A Very Radio Loud, Narrow-Line Quasar with Relativistic Jets? ApJ, 584 (2003) 147. 